Cahiers d'études africaines

\title{
Viol d'hommes, masculinités et conflits armés
}

Male Rape. Masculinities and Armed Conflicts

\section{Marc Le Pape}

\section{CpenEdition}

\section{Journals}

Édition électronique

URL : http://journals.openedition.org/etudesafricaines/17290

DOI : 10.4000/etudesafricaines. 17290

ISSN : 1777-5353

\section{Éditeur}

Éditions de l'EHESS

\section{Édition imprimée}

Date de publication : 28 mai 2013

Pagination : 201-215

ISBN : 978-2-7132-2387-7

ISSN : 0008-0055

\section{Référence électronique}

Marc Le Pape, "Viol d'hommes, masculinités et conflits armés », Cahiers d'études africaines [En ligne], 209-210 | 2013, mis en ligne le 06 juin 2015, consulté le 15 juin 2020. URL : http://

journals.openedition.org/etudesafricaines/17290 ; DOI : https://doi.org/10.4000/etudesafricaines. 17290 


\section{Viol d'hommes, masculinités et conflits armés}

Nombre d'enquêtes et de publications ont été consacrées aux viols d'hommes et d'adolescents en temps de paix dans les prisons et des institutions religieuses. Par contraste les mêmes actes commis dans les situations de conflits armés n'ont suscité un champ d'études qu'à une date relativement récente. Cependant, plusieurs travaux historiques ont évoqué les viols d'hommes en temps de guerre, notamment ceux rassemblés sous la direction de R. Branche et F. Virgili (2011), c'est en particulier le cas d'une recherche portant sur « les hommes oubliés » de la guerre de libération du Bangladesh (Mookherjee 2011 : 71-81). À l'occasion de leurs travaux sur des cas récents, les juristes S. Sivakumaran (2007 : 257-258) et L. Stemple (2009 : 612-613) dressent une liste de conflits armés où ces violences ont été repérées, tous deux soulignent leur fréquence au cours des conflits armés. La période retenue ici pour engager l'observation débute avec les guerres en ex-Yougoslavie, soit au commencement des années 1990. En effet, ce sont ces guerres et les travaux du Tribunal pénal international pour l'ex-Yougoslavie (TPIY), créé en 1993, qui ont provoqué l'émergence d'une attention collective sur tous les crimes sexuels, y compris ceux déclarés par des victimes hommes ${ }^{1}$.

Les juristes du TPIY et les activistes du droit qui les entouraient pour influencer leurs pratiques ont obtenu que les violences sexuelles (y compris celles contre les hommes) soient jugées en tant que telles et non pas exclusivement lorsqu'elles interviennent au cours de tortures comme par exemple dans les cas décrits à propos de la guerre d'indépendance algérienne par l'historienne R. Branche (2001 : 331-334) et dénoncés par J.-P. Sartre (1964 : $77-81)^{2}$, par P. Bourdieu (2002 : 38-39) également lorsqu'il évoque des tortures délibérément organisées en vue de féminiser des prisonniers, de les humilier sexuellement.

Sous le terme de violences sexuelles contre des hommes lors de conflits armés, le juriste S. Sivakumaran (2007 : 261-267), prenant appui sur le statut

1. Pour nos discussions et sa lecture critique de ce texte, je tiens à remercier Claudine Vidal.

2. SARTRE (1964 : 77-81) commente La Question que vient de publier Henri Alleg (1958). Parmi les tortures subies par H. Alleg, des actes qui aujourd'hui tomberaient sous le chef de violences sexuelles, notamment la nudité forcée : on l'attachait nu à une planche, « on lui brûlait les seins, les poils du sexe ». 
de la Cour pénale internationale, comprend le viol (oral et anal), la stérilisation forcée (castration et autres formes de mutilations), la nudité et la masturbation forcées, les coups sexuellement orientés.

Je montrerai tout d'abord que la question de ces agressions sexuelles n'a été publiquement posée que grâce à l'engagement d'un certain nombre d'acteurs, journalistes, chercheurs, praticiens humanitaires. Il apparaîtra ensuite que diverses conceptions de la masculinité ont fait obstacle à cette prise en compte. Enfin, je présenterai un ensemble de publications consacrées aux conflits armés à l'Est de la République démocratique du Congo (RDC) où apparaissent les coopérations entre chercheurs, journalistes et acteurs d'ONG.

\section{L'émergence de la question}

Le New York Times du 5 août 2009 publie un reportage effectué à Goma et Bukavu en RDC : le journaliste rappelle que les conflits armés à l'Est du Congo qui n’ont pas cessé, dans les provinces du Kivu, depuis au moins 1996 sont connus pour le nombre considérable des viols de femmes qui y ont été commis au cours des années 2000. Cependant l'auteur de cet article, J. Gettleman (2009), met l'accent sur une caractéristique de ces conflits jusqu'alors peu médiatisée, les viols d'hommes dont il souligne une augmentation sévère en 2009 avec le développement d'importantes opérations militaires. En témoignent des organisations internationales de défense des droits humains, des organisations de secours médical, des hommes victimes - quatre d'entre eux ont accepté que leur photographie soit placée en tête de l'article, alors même que, selon Gettleman, dans une région où l'homosexualité est un «tabou », ces viols affectent les victimes d'une dose supplémentaire de honte ( carry an extra dose of shame »). Dans la suite de ce reportage, un second événement de presse doit être relevé : le 17 juillet 2011, The Observer, à Londres, fait paraître un long article intitulé «Le viol d'hommes » (Storr 2011). Il s'agit d'une enquête conduite à Kampala (Ouganda) dans un centre d'assistance aux réfugiés parmi lesquels des Congolais victimes de viols liés au conflit armé en RDC; le journaliste souligne qu'il a pu rencontrer quelques hommes acceptant de témoigner grâce à la médiation du médecin anglais responsable de cette institution, le docteur Chris Dolan. Pour attester la fréquence de ces abus sexuels W. Storr se réfère à la seule enquête épidémiologique qui les ait pris en compte et ait évalué leur prévalence : $22 \%$ des hommes interrogés déclarent avoir été victimes des divers types de violences sexuelles liées aux conflits armés dans l'Est de la RDC (Ituri, Sud et Nord Kivu), entre 1994 et 2010 - 4,4 \% déclarent avoir été victimes de viols (Johnson \& Scott 2010). Il reste que le médecin responsable de ce centre d'assistance constate, en le regrettant, que les ONG travaillant sur la violence liée au genre ( sexual and genderbased violence ») 《font systématiquement silence » sur ces agressions contre les hommes, ou «les mentionnent incidemment à la fin d'un rapport ». 
L'attention aux violences sexuelles concernant les hommes ne date pas des conflits affectant la RDC. En effet, le Tribunal pénal international pour l'ex-Yougoslavie (TPIY), créé en 1993, a eu à juger de nombreux cas où des hommes se déclaraient victimes d'agressions sexuelles (Campbell 2007); pourtant, en 1997, la revue médicale britannique The Lancet publiait un courrier signalant à la fois le manque d'assistance médicale spécifiquement adressée à ces victimes et les cruautés physiques dont il était question : «Des prisonniers ont été forcés de violer d'autres prisonniers, ont été forcés à pratiquer des fellations sur leurs gardes et entre eux, ont subi des castrations et circoncisions ainsi que d'autres mutilations » (Stener Carlson 1997). Ainsi, tandis que le Tribunal pénal international pour le Rwanda (TPIR) a reconnu en octobre 1998, lors du procès Akayesu, que le viol était constitutif du crime de génocide (Lewis 2009 : 28-34), au TPIY « le chef de viol, reconnu comme crime contre l'humanité dans le statut [du TPIY], fut étendu par la jurisprudence aux violences sexuelles » (Jurovics 2009 : 59). Cette jurisprudence fut appliquée aux agressions sexuelles à l'encontre des hommes, devenues ainsi condamnables en tant que telles, et non plus sous le chef de tortures, traitements cruels et actes inhumains. Il reste, qu'à la différence des viols exercés contre les femmes en Bosnie puis sur plusieurs terrains de guerre en Afrique, viols qui ont suscité une profusion d'études émanant de plusieurs disciplines académiques, qui ont été fréquemment médiatisés et sont devenus un champ d'intervention privilégié par les ONG humanitaires et leurs bailleurs de fonds, les violences sexuelles infligées aux hommes, durant les années 1990, n'ont fait l'objet, dans un premier temps, que de rares études particulières et n'ont été qu'exceptionnellement reconnues par des journalistes (Gutman 1994 : 223).

Lors d'un colloque tenu en 2002, deux intervenants citent une enquête portant sur 4076 ONG s'occupant de violences sexuelles en temps de guerre et de crises politiques : ils relèvent que seules $3 \%$ d'entre elles mentionnent dans leurs programmes un intérêt pour des hommes victimes. En outre sur ces 4076 oNG, seules 60 apportent, en situations de conflit armé, une assistance directe aux individus sexuellement agressés, les autres interviennent par des plaidoyers ou comme think-tanks. Enfin 58 de ces 60 oNG opérationnelles précisent que les victimes d'agressions sexuelles sont des «femmes et/ou des jeunes filles », et 2 usent du terme générique de «personnes ». Néanmoins les auteurs de cette communication citent un rapport de l'Organisation mondiale de la santé (2000) soulignant que, durant les conflits armés, des violences sexuelles sont exercées contre «les adultes mâles, les adolescents et les jeunes garçons »; le risque de viol est accentué dans les centres de détention (DelZotto \& Jones 2002 : 5-6).

Ainsi, à la différence des viols d'hommes commis en prison qui, de longue date, ont fait l'objet d'enquêtes, de mesures statistiques, de récits et d'études (Brownmiller 1975 : 313-326 ; Sivakumaran 2005 : 1299-1304 ; Stemple 2009 : 608-611), la généralisation de l'intérêt à l'égard des violences sexuelles concernant les hommes en temps de conflits armés n'est observable 
qu'après la création des Tribunaux ad hoc (TPIY et TPIR) en 1993 et 1994 et il aura donc fallu plus de quinze ans pour que cette question soit traitée par des articles publiés dans des journaux de stature internationale, tels The New York Times et The Observer. Dans ce laps de temps, se multiplient les publications émanant de juristes, de politistes, d'épidémiologues, ainsi que les rapports produits par des chercheurs travaillant dans le cadre d'ONG, d'Agences des Nations Unies et du Comité international de la Croix-Rouge $(\mathrm{CICR})^{3}$.

\section{Le thème de la masculinité}

Au cours des années 2000, le thème de la masculinité est constamment abordé par les auteurs qui, traitant des violences de guerre d'ordre sexuel, évoquent celles qui affectent les hommes. La masculinité est envisagée sous l'angle du statut, de la représentation et du vécu traumatique. Un cliché illustrant le long article de W. Storr publié par The Observer en juillet 2011 synthétise de façon dramatique ces trois aspects de la masculinité brisée par la violence sexuelle subie. La légende de la photographie précise qu'il s'agit d'un Congolais, victime de viol : il réside en Ouganda, «sa femme l'a quitté car elle ne pouvait accepter ce qui est arrivé », il a tenté de se suicider fin 2010. L'homme est assis sur un matelas. Il est recroquevillé, la tête posée sur les genoux et enfouie dans ses bras. Il veut rester anonyme. Le cliché n'est pas un gros plan sur l'homme assis. Il le montre dans une chambre dont les murs sont nus, le matelas est posé sur le sol, il y a une moustiquaire suspendue au plafond, une télévision éteinte sur une tablette contre le mur, un thermos. Très peu d'objets, rien de personnel.

Le journaliste prend appui, dans son article, sur une recherche de l'universitaire et juriste L. Stemple (2009) ; celle-ci montre que les violences sexuelles contre les hommes sont courantes dans de nombreux conflits armés et que l'aide internationale échoue à les prendre en charge. Sur ce dernier point, W. Storr fait état d'un échange de mail avec Lara Stemple qui maintient et même accentue son jugement : le droit humanitaire international n'a pas pris en compte les violences sexuelles frappant les hommes; elle évoque ainsi la résolution 1325 du Conseil de Sécurité des Nations Unies consacrée exclusivement aux « effets des conflits armés sur les femmes et les petites filles » (octobre 2000). Selon Stemple, en raison de ce désintérêt à l'égard des hommes victimes, la résolution 1325 nuit aux femmes elles-mêmes en les assignant systématiquement au statut de victime et en attribuant simultanément aux hommes une soi-disant invulnérabilité. Sans affirmer que ces brutalités à l'encontre des hommes adviennent dans toute guerre, Stemple

3. S. Sivakumaran (2007: 257-258) et L. Stemple (2009: 612-613) dressent chacun une liste des conflits armés à propos desquels ces violences contre des hommes ont été documentées. 
ajoute qu'il n'est pas risqué de dire qu'elles existent dans la plupart des conflits armés et que le tabou sur ce type d'actes explique en partie le silence dans lequel ils sont tenus.

L'article de Will Storr se présente comme un reportage classique : le journaliste s'est rendu à Kampala pour enquêter sur les hommes victimes de violences sexuelles en temps de guerre. Il en a rencontré plusieurs, retrace le parcours biographique de l'un d'entre eux et relate les circonstances des viols subis par cet homme, s'entretient longuement avec des soignants. L'article a un caractère militant : son auteur cherche à faire reconnaître le problème du tabou pesant sur ces violences spécifiques. Cependant, l'originalité de ce travail journalistique tient à son association avec une recherche universitaire, celle de Lara Stemple à laquelle il se réfère à plusieurs reprises et dont il retient le point de vue pour conclure son article. L'enquête auprès des soignants conclut sur l'affirmation que ces hommes violés doivent être écoutés et soignés par des spécialistes préparés à reconnaître l'étiologie spécifique de leurs blessures corporelles ainsi que de leur dépression, préparés à poser un diagnostic qui n'efface pas le fait du viol.

L'un des témoins cité par W. Storr raconte qu'il a caché à son propre frère les raisons pour lesquelles il consultait dans un centre de soins : « J'ai peur qu'il me dise : "Maintenant mon frère n'est pas un homme”. » Le sentiment individuel de «perte» du statut masculin, pour dramatique qu'il soit, est aggravé par d'autres risques: d'une part, le rejet par ses proches et son milieu familial, d'autre part, le danger d'être traité d'homosexuel d'où l'éventualité, en Ouganda, d'être poursuivi en justice, ce qui est, selon le journaliste, le cas dans 38 pays africains sur 53.

Le travail de la juriste L. Stemple (2009), sur lequel W. Storr prend appui, a pour but principal d'analyser les instruments juridiques dont disposent les tribunaux et les activistes des droits humains dans les cas de violences sexuelles contre les hommes. Elle souligne que l'approche en termes de genre a conduit les ONG internationales de secours et de droits de l'homme, ainsi que les agences des Nations Unies, à privilégier une approche focalisée sur les violences sexuelles contre les femmes, légitimant ainsi une hiérarchie de ces violences situant les hommes adultes, qui en sont victimes, au bas de cette hiérarchie. En arrière-plan de cette hiérarchie, elle reconnaît l'influence des représentations les plus courantes de la masculinité qui conduisent à accepter le stéréotype selon lequel un «vrai » homme, par sa résistance, rendrait le viol impossible. D'où aussi la rareté avec laquelle les hommes se reconnaissent victimes en raison du risque d'apparaître comme un «mâle féminisé » et comme homosexuel. Sur ce point, elle conteste la manière dont, en 1975, une journaliste féministe, Suzanne Brownmiller, dans son livre pionnier, rendait compte du viol comme « un acte d'intimidation par lequel tous les hommes gardent toutes les femmes dans un état de peur ». La catégorie des coupables est ainsi construite comme homogène du point de vue du genre, elle comprend potentiellement tous les hommes : violeurs en général, ils ne peuvent être perçus comme victimes de violences 
sexuelles. L. Stemple montre que ce type d'énoncés, propre à Brownmiller et au féminisme des années 1970, a, au moins jusqu'aux années 1990, influencé nombre d'institutions de secours et d'organisations internationales des droits - il reste actuellement toujours influent. Pour critiquer la catégorisation produite par Brownmiller, Stemple prend appui sur de nouveaux courants féministes et queer en se référant notamment à Alice M. Miller et Judith Butler. Elle contribue ainsi à une mise en cause de cette perception normative de la masculinité comme naturellement inviolable d'où il résulte que les femmes sont quant à elles assignées à une identité de faible victime.

La critique de l'approche féministe héritée de Brownmiller avait déjà été conduite au début des années 2000 dans le cas spécifique de son application aux conflits armés, en particulier ceux de l'ex-Yougoslavie. Ainsi, DelZotto et Jones, en 2002, ont-ils mené cette critique d'une conception étroite de la masculinité associée à une conception non moins étroite de la victimisation des femmes. Ces auteurs soulignent l'influence pratique de ce cadre de pensée qui a conduit, dans la plupart des conflits des années 1990 et 2000, à ne délivrer des secours qu'aux femmes et jeunes filles victimes de violences sexuelles, sauf quelques exceptions. Ils contribuent en outre à donner un éclairage historique et politique à ce cadre de pensée et d'action. Analysant des documents du Congrès et du Département d'État américains, ainsi que des sources parlementaires, ils affirment qu'à la fin de la guerre froide les États-Unis visaient à se construire un rôle de protecteur allant au secours des femmes en détresse, sexuellement agressées par des hommes barbares. Ce fut un thème de légitimation des interventions diplomatiques et militaires dans certaines régions, tirant parti de la reconnaissance institutionnelle des crimes de guerre sexuels (contre les femmes) : ceux-ci sont exploités en faveur d'une stratégie d'intervention, notamment par le biais du financement d'ONG dont l'action doit se porter spécifiquement au secours des femmes. Selon ces auteurs, cette stratégie a puissamment contribué à occulter les victimes sexuelles masculines. En 2002, dans le champ universitaire, cette critique politique était novatrice.

Le juriste S. Sivakumaran (2005, 2007), auquel se réfère fréquemment L. Stemple, place l'analyse de la masculinité à l'épreuve des conflits armés au cœur de ses travaux. En 2005, cherchant à comprendre pour quelles raisons les viols d'hommes ont été si peu pris en considération au cours des affrontements armés contemporains (c'est-à-dire durant les années 1990 et 2000), il détermine quels agents sont producteurs de ces restrictions culturelles et politiques. En premier lieu, il met en cause le mouvement des femmes et le mouvement homosexuel (qu'il dénomme aussi mouvement queer), dont on aurait pu escompter qu'ils prennent en compte ces violences, mais qui ne l'ont pas fait. La conception féministe du viol comme acte de domination masculine sur les femmes est associée, chez une juriste américaine comme Catharine MacKinnon, au constat selon lequel le viol dépouillerait l'homme de son statut masculin, dégraderait sa masculinité, en le plaçant dans un rôle 
de femme ${ }^{4}$. Cette affirmation fréquemment admise et émanant d'une féministe influente n'a pourtant pas eu pour effet de constituer ces viols en cause du mouvement des femmes. Sivakumaran s'en déclare surpris et exprime son désaccord. Il fait un constat analogue pour le mouvement homosexuel américain et identifie trois raisons à son non-engagement. La première tient à l'argument selon lequel il existe un préjugé social pour lequel le viol d'hommes par des hommes ne se produit qu'entre homosexuels. Dans ce contexte, une mobilisation du mouvement gay en faveur des victimes masculines en situation de conflit armé risquerait de renforcer le préjugé selon lequel les violeurs d'hommes et les victimes de ces viols sont par nature homosexuels. En outre, étant donné la faible audience internationale du mouvement gay, ses activistes choisissent de privilégier la lutte pour l'égalité et la non-discrimination (Sivakumaran publie en 2005, son point de vue devrait être aujourd'hui nuancé ${ }^{5}$, du moins quant aux discours tenus). Enfin, ces activistes ne s'occupent de victimes masculines de viols que lorsque ces attaques ont pour origine la haine des homosexuels. Finalement, la convergence entre les mouvements féministes et queer en faveur des violences sexuelles subies par des hommes n'a pas eu lieu. Pourtant Sivakumaran (2005 : 12801285) estime qu'elle est politiquement cohérente car les deux mouvements ont un intérêt commun à lutter contre les stéréotypes de genre qui fondent les formes dominantes de la masculinité.

Plus généralement, c'est, pour Sivakumaran, la force du préjugé homophobe qui conduit à ne pas s'occuper de ces viols : en effet ce préjugé est associé, par le sens commun, à la conviction qu'il y a un lien entre ces viols et l'homosexualité. Sivakumaran adopte trois perspectives pour décrire cette configuration singulière : sa description porte sur le langage, l'expérience des victimes, l'intention du violeur. En ce qui concerne le langage, Sivakumaran insiste sur l'indétermination qui existe dans les discours entre viol homosexuel ("homosexual rape ») et viol entre hommes ("male/male rape »), la confusion entre les deux s’accordant avec les perceptions homophobes qui ne prennent ainsi pas en compte la violence des agresseurs «en majorité hétérosexuels ». Ce point concerne particulièrement les affaires de viols dans les prisons. Le silence tient aussi aux constructions sociales dominantes : selon celles-ci, les hommes ayant subi des violences sexuelles, n'ayant pu se défendre, ont perdu leur masculinité, voire sont entachés d'homosexualité,

4. Éric FASSIN (2009: 171-172), dans son étude sur le date rape aux États-Unis, indique que selon C. MacKinnon, «figure emblématique du féminisme culturel », dans les conditions de domination masculine, « le rapport sexuel doit être compris comme un viol » : « dans un rapport dominé, le consentement n'est qu'une illusion » (l'expression date rape "se traduit malaisément», elle désigne les agressions masculines dans le contexte du rendez-vous amoureux).

5. Voir, notamment, la déclaration du secrétaire général des Nations Unies en 2012, Centre d'actualités de l'ONU, 7 mars 2012, «L'ONU appelle les États à s'attaquer à la violence liée à l'orientation sexuelle », <http://www.un.org/apps/newsFr/ storyF.asp?NewsID=27741\&Cr=orientation\%20sexuelle \&Cr1=\#.UEnP-ESTOr8>. 
ce qui les décourage de s'exprimer pour ne pas paraître gay ( straight victims don't want to appear gay »). Quant à l'intention du violeur, elle consiste à placer la victime dans une situation que l'agresseur estime caractéristique de l'homosexualité.

Dans un second article spécifiquement consacré aux violences dans les conflits armés, Sivakumaran (2007 : 261-267) reprend ses analyses précédentes tout en dressant un tableau plus minutieux des différentes formes de violence sexuelle dont il établit une typologie conforme à celle de la Cour pénale internationale (CPI) telle qu'elle est présentée dans le statut de Rome. Enfin, il s'appuie sur un article de Adam Jones (2006) pour préciser le contenu du stéréotype de la masculinité auquel il se réfère: il s'agit du «standard hétéronormatif » car «le concept de masculinité hégémonique renvoie au mâle hétérosexuel ».

\section{Masculinités et hommes victimes à l'Est du Congo (RDC)}

Entre 2006 et 2009, deux chercheuses suédoises, M. Eriksson Baaz et M. Stern, ont réalisé une enquête auprès de 2226 officiers et soldats appartenant aux Forces armées congolaises (FARDC) et dont $80 \%$ d'entre eux ont été ou sont présents dans les zones de conflit du Nord et du Sud Kivu. Elles ont utilisé une méthode d'entretiens semi-directifs avec des petits groupes de trois à quatre militaires. La première partie des entretiens portait sur les qualités du bon soldat, la seconde partie concernait leur approche de la masculinité et de la féminité en relation avec le statut de combattant, l'entretien s'achevait par une discussion sur la violence sexuelle. Aucun des soldats ne reconnut avoir commis des abus sexuels, néanmoins « la manière dont la plupart parlaient spécifiquement de cas de viols montrait qu'ils parlaient d'expériences personnelles, ou tout au moins familières » (Eriksson Baaz \& Stern 2010 : 10). Caractéristique rare de ce travail académique en RDC, il prend en compte les points de vue de violeurs potentiels. Les soldats en majorité attribuent l'incidence du viol à leur pauvreté et à la pénibilité de leur condition de vie. Selon un officier « le soldat qui n'a pas d'argent, ne passera pas par la voie normale pour obtenir ce qu'il veut. Il ne peut ni manger, ni boire, n'a rien à donner à une femme, il la prendra par la force. Physiquement, les hommes ont des besoins. Ils ne peuvent rester longtemps sans être avec une femme ». Il conclut que si le soldat avait de l'argent cela réduirait considérablement la fréquence des viols (ibid. : 31). Les enquêtrices font remarquer que beaucoup d'hommes sont pauvres sans nécessairement se conduire en violeurs. C'est la combinaison de la masculinité militaire placée en situation de conflit armé avec la pauvreté et la relative impunité des violeurs qui contribue aux violences physiques des soldats contre les personnes, notamment au moment de pillages. Comme d'autres études et rapports consacrés au Kivu, depuis les cinq dernières années, M. Eriksson Baaz 
et M. Stern insistent sur l'exposition des hommes et des garçons aux violences de genre (Gender Based Violence, GBV) et la longue méconnaissance qui a rendu ces crimes « invisibles ${ }^{6}$.

Lors de son enquête en Ouganda, W. Storr avait interviewé le docteur Chris Dolan car ce dernier était responsable d'un centre d'accueil où des Congolais victimes de violences sexuelles étaient pris en charge. Or ce médecin a dirigé une enquête conduite sur quatre sites au Nord et au Sud Kivu et portant sur les perceptions locales de la violence sexuelle. En outre, le rapport d'enquête, publié en novembre 2010, se réfère aux entretiens avec des acteurs institutionnels congolais et internationaux travaillant sur ce type de violence (Dolan 2010). L'un des problèmes sur lequel le rapport insiste est celui de la rareté des données chiffrées fiables permettant d'apprécier de manière rigoureuse la mesure des violences sexuelles en particulier contre des hommes. Comme dans la plupart des travaux précédemment cités, le thème de la honte et donc du silence de ces victimes explique la difficulté d'établir des données chiffrées (ibid. : 52-54); le phénomène de sous-déclaration est donc général de la part des victimes, d'où une sous-estimation systématique de ces violences spécifiques. La crainte de subir des représailles par l'entourage ainsi que la quasi inexistence de secours spécifiques accentuent la sousdéclaration - en effet ceux qui se déclarent le font généralement pour accéder à des soins, il leur faut donc surmonter la crainte d'être mal reçu par un personnel médical non spécialisé. De fait, jusqu'en 2010, deux évaluations sont régulièrement citées comme mesure des violences sexuelles contre les hommes. L'une est produite par Médecins sans frontières en 2009, indiquant que « approximativement \% $\%$ des victimes de viols venant en consultation dans l'hôpital de Masisi (Nord-Kivu) sont des hommes (MSF 2009 : 11). La deuxième estimation provient de l'American Bar Association North Kivu qui, par rapport à l'ensemble des personnes qui s'adressent à ses services, estime à $10 \%$ l'incidence des violences sexuelles contre les hommes (Eriksson Baaz \& Stern 2010 : 44). L'étude épidémiologique sur la violence sexuelle à l'Est de la RDC, publiée en août 2010 par The Journal of the American Medical Association, constitue le premier article tentant d'établir une mesure épidémiologique des agressions à la fois contre les femmes et les hommes.

6. La politiste S. AUTESSERRE (2012 : 213-218), dans un article sur les violences à l'Est du Congo, montre que trois récits dominent le discours public actuel sur la RDC et ont une influence contreproductive par rapport à leurs propres intentions. Le premier porte sur l'exploitation illégale des ressources minérales, le deuxième concerne le viol, le troisième a pour thème l'idée que la reconstruction de l'État serait le moyen le plus efficace pour mettre fin aux abus sexuels et à l'exploitation illégale des ressources naturelles. Elle relève que l'énorme concentration, nationale et internationale, de l'intérêt sur les abus sexuels dont les femmes sont victimes provoque une discrimination en termes d'aide à l'égard des autres populations vulnérables, et en particulier à l'égard des hommes eux aussi victimes d'agressions sexuelles. 
Les conclusions de cet article sont de deux ordres : elles relèvent la minimisation par la communauté internationale d'une part du nombre d'hommes victimes de violences sexuelles et, d'autre part, du nombre de femmes auteures de brutalités sexuelles et de violation des droits humains.

Les études dont il vient d'être rendu compte se réfèrent couramment à des préjugés et des stéréotypes généraux concernant les masculinités dont elles affirment l'influence déterminante en particulier sur la perception des hommes victimes d'exactions sexuelles. Il reste que les auteurs ne présentent pas en détail les données empiriques sur lesquelles ils s'appuient pour identifier ces préjugés. C'est pourquoi il semble éclairant d'analyser brièvement les commentaires suscités par l'article que W. Storr a consacré, en 2011, dans The Observer, aux crimes sexuels contre des hommes commis durant des conflits armés dans les provinces du Kivu. En réaction à l'article, 209 commentaires ont été publiés par The Observer entre le 17 et le 19 juillet 2011. Je ne prétends nullement mener une enquête d'opinion qui aurait une base statistique. C'est plutôt dans une intention monographique que je me suis intéressé à décrire ce petit événement de presse qui constitue un appendice à l'article de W. Storr : que disent certains lecteurs et lectrices qui ont pris la peine d'écrire des commentaires et ont débattu entre eux ? Ils témoignent d'interrogations sur le traitement des violences sexuelles subies par des hommes en temps de guerre: sont-elles reconnues ou niées par les organisations et institutions engagées dans l'aide aux victimes de conflits armés ? Fait-on preuve de misogynie et d'hostilité au féminisme lorsque l'on dénonce l'usage restrictif de la notion de genre (il s'agirait d'hostilité à un courant féministe, "the old feminist view ») ? Comment expliquer la stigmatisation des victimes, le silence sur leur sort social, les résistances à leur prise en charge ? Comment expliquer le choix de violer des hommes, s'agit-il de « viols homosexuels », d' « homosexualité latente », ou bien doit-on imputer ces violences à la situation de conflit armé, et les considérer exclusivement comme des actes de pouvoir, une volonté d'humilier, de terroriser, démontrer sa puissance, faire souffrir ("rape is about power») ? Comment organiser l'aide, notamment médicale, à ces hommes, doit-elle être distincte, différente de l'assistance portée aux femmes ? Je résume aussi fidèlement que possible les principaux thèmes, tels qu'ils sont exprimés par les rédacteurs de commentaires.

Nombre de lecteurs cherchent à comprendre la conduite des violeurs, à énoncer leurs motivations, ce qui ne signifie en rien et pour aucun les excuser. Tout au long des 209 messages, deux courants s'opposent ; les uns affirment que la motivation sexuelle et la recherche du plaisir sont déterminantes tandis qu'un second courant considère que, en situation de violences armées comme c'est le cas à l'Est de la RDC, les viols ont pour motif la volonté de terroriser, d'humilier, de blesser ce qui n'a rien à voir avec le « désir » ou « la sexualité ». Ces deux camps restent sur des positions tranchées et inconciliables. À ce débat vient se greffer la discussion sur une hypothétique association entre les viols d'hommes par des hommes et une « homosexualité 
latente » du violeur et du violé. Face à ce point de vue, plusieurs rétorquent que, dans un contexte d'homophobie et de domination des normes hétérosexuelles, il est aberrant d'attribuer l'acte de viol, en situation de conflit armé, à une motivation homosexuelle. D'une part c'est réduire l'homosexualité à un acte (violent en l'occurrence), d'autre part c'est ignorer la prédominance et la pression d'une «culture macho », à laquelle il est «nécessaire de mettre fin » ("Let's do what ever is necessary to stop rape culture and put an end to machismo »). Il ne s'agit pas ici de départager ces points de vue.

À plusieurs reprises, des lecteurs reprennent à leur compte les critiques adressées aux agences des Nations Unies et aux ONG internationales : ces commentaires contestent les points de vue qui, identifiant les rapports de genre à un rapport de domination des hommes sur les femmes, en déduisent que seules les femmes peuvent être victimes tandis que les hommes sont exclusivement du côté de l'exercice de la violence. Ainsi un post rappelle que certaines féministes radicales des années 1980 désignaient les hommes « comme une classe d'oppresseurs », point de vue qui conduit à réserver aux femmes le statut de victimes et aux hommes celui d'auteurs des violences ( "perpetrators $»)$. Cependant, nombre de messages contestent et dénoncent le blâme contre « les féministes » : celles-ci ont ouvert la voie à la dénonciation publique de la violence sexuelle, elles ont combattu le tabou qui empêchait le déploiement de la critique, et la prise de parole sur les viols d'hommes en temps de guerre prend appui sur ce travail accompli par des féministes. Un commentaire de Michael Amherst $(2010)^{7}$ reprend la réflexion sur les politiques de genre adoptées par la plupart des organisations d'assistance : ce qui est critiquable ce n'est pas le féminisme en général mais une conception binaire du genre selon laquelle l'acte de viol serait « une conduite purement masculine », les hommes se caractérisant par « une sexualité agressive » tandis qu' "être victime est considéré comme un signe de féminité et de faiblesse ». Ce sont donc ces notions binaires du genre qui devraient être démantelées. Plusieurs commentaires rappellent que, dans les zones de conflit, les victimes de violences sexuelles sont massivement des femmes; aucun d'entre eux ne contredit ce constat mais beaucoup cependant se déclarent surpris par l'importante proportion d'hommes-victimes dans les zones de conflit à l'Est de la RDC.

\section{Recherches et plaidoyer}

Il existe une réflexion analytique et théorique considérable sur la question des rapports entre genre, sexualité et violence ; ces travaux sont imbriqués dans des controverses complexes et sophistiquées sur «le sexe politique »,

7. M. Amherst intervient à plusieurs reprises dans le débat entre commentateurs du reportage de W. Storr, en particulier lorsqu'il s'agit de critiquer l'influence d'un courant féministe issu des thèses de Brownmiller. 
«le trouble dans le genre », l'ordre « hétéronormatif», etc. De la plupart de ces travaux, les conflits armés sont absents, sauf à l'occasion de quelques conflits où la violence sexuelle fut jugée ou exhibée - ainsi J. Butler (2010 : 79-96) intervient-elle lorsque cette violence fut jugée mais d'abord exhibée avec la diffusion des photographies prises par des Américains dans la prison d'Abou Ghraïb en Irak et montrant les sévices commis par le personnel américain de surveillance : "Il y a des photos de corps liés ensemble, d'individus tués, de fellations forcées, de dégradations déshumanisantes, et ces photos ont été prises sans entraves »; la photo d'une gardienne tenant un prisonnier irakien par une laisse fit la Une du New York Times.

Pour leur part, les chercheurs, qui se consacrent à l'étude des conflits armés, ont régulièrement évoqué, analysé l'articulation entre combats et violences sexuelles contre la population civile féminine. De nombreuses enquêtes ont confirmé l'importance numérique de ces viols et en ont montré leurs terribles conséquences sur la vie des femmes. Dans la plupart des situations de guerre actuelles et récentes, la fréquence des agressions sexuelles contre les femmes a été mesurée et ces mesures, qui attestent leur intensité, ont été médiatisées.

Cependant plus rares sont les auteurs qui ont orienté leur attention sur les agressions sexuelles contre des hommes, non combattants, adversaires vaincus, captifs, combattants tués et mutilés. Ces auteurs, quant à eux, n'ignorent nullement les controverses entre analystes des rapports de genre, de la multiplicité des masculinités, de la norme hétérosexuelle : ils évoquent ces controverses parfois pour indiquer quels partis ils en tirent mais aussi pour souligner en quoi elles ont contribué à rendre leur objet invisible, à instituer une hiérarchie d'intérêts de recherche minimisant les investigations qui ne se focalisaient pas sur les victimes féminines. Au cours des années 2000 s'est néanmoins développé un courant d'études et de recherches dont il a été ici partiellement rendu compte. Les auteurs universitaires sont très majoritairement anglophones (américains principalement, mais aussi anglais, canadiens, suédois). Ce sont principalement des juristes et des politistes, la plupart ayant eu d'abord un parcours de praticiens, soit auprès de tribunaux, soit sur un terrain de conflits en travaillant pour une ONG ou une agence des Nations Unies. Après quoi ces praticiens-chercheurs ont généralement intégré des institutions universitaires tout en continuant à intervenir en tant qu'experts auprès d'organisations internationales. Quelle que soit leur perspective professionnelle d'observation et leurs terrains, les chercheurs dont les travaux ont été ici évoqués se sont tous engagés dans un plaidoyer pour que les violences spécifiques qu'ils étudiaient soient identifiées et jugées, pour qu'elles soient prises en charge par une assistance médicale et une attention aux paroles de souffrance, pour enfin que les mesures épidémiologiques et statistiques de ces violences soient développées afin que leur réalité et leur ampleur ne soient plus méconnues, voire contestées. 


\section{BIBLIOGRAPHIE}

AlleG, H.

1958 La Question, Lausanne, Éditions La Cité.

AmHERST, M.

2010 « Rape is Not Just a Women's Isssue », The Guardian, 17 mars 2010.

AutesserRe, S.

2012 «Dangerous Tales : Dominant Narratives on the Congo and Their Unintended Consequences », African Affairs, 111 (443) : 202-222.

Bourdieu, P.

2002 La domination masculine, Paris, Éditions du Seuil.

BRANCHE, R.

2001 La torture et l'armée pendant la guerre d'Algérie 1954-1962, Paris, Gallimard.

2002 « Des viols pendant la guerre d'Algérie », Vingtième siècle. Revue d'histoire, $75: 123-132$.

BRANChe, R. \& VIRGILI, F. (DIR.)

2011 Viols en temps de guerre, Paris, Payot.

BROWNMILLER, S.

1975 Against Our Will. Men, Women and Rape, New York, Simon \& Schuster [trad. française, Le Viol, Paris, Stock, 1976].

BUTLER, J.

2010 Ce qui fait une vie. Essai sur la violence, la guerre et le deuil, Paris, ZonesLa Découverte.

CAmpBell, K.

2007 «The Gender of Transitional Justice : Law, Sexual Violence and the International Tribunal for the Former Yugoslavia », The International Journal of Transitional Justice, 1 (3) : 411-432.

DelZotto, A. \& Jones, A.

2002 Male-on-Male Sexual Violence in Wartime: Human Rights' Last Taboo?, Paper presented to the Annual Convention of the International Studies Association (ISA), New Orleans, LA, 23-27 March.

Dolan, C.

2010 «War Is Not Yet Over». Community Perceptions of Sexual Violence and its Underpinnings in Eastern DRC, London, International Alert.

Eriksson BAaz, M. \& STERn, M.

2010 The Complexity of Violence. A Critical Analysis of Sexual Violence in the Democratic Republic of Congo (DRC), Uppsala, The Nordic Africa Institute. 
FASSIN, É.

1997 «Le date rape aux États-Unis. Figures d'une polémique », Enquête, 5 : 193222, <http://enquete.revues.org/document1263.html>.

2009 Le sexe politique. Genre et sexualité au miroir transatlantique, Paris, Éditions de l'EHESS.

Gettleman, J.

2009 «Symbol of Unhealed Gongo : Male Rape Victims », The New York Times, August 5, <http://www.nytimes.com/2009/08/05/world/africa/05congo.html>.

GutMan, R.

1994 Bosnie : témoin du génocide, Paris, Desclée de Brouwer.

Johnson, K. \& ScotT, J.

2010 «Association of Sexual Violence and Human Rights Violations with Physical and Mental Health in Territories of the Eastern Democratic Republic of the Congo », Journal of the American Medical Association, 304 (5) : 553-562.

JONES, A.

2006 « Straight as a Rule. Heteronormativity, Gendercide, and the Noncombatant Male », Men and Masculinities, 8 (4) : 451-469.

JUROVICS, Y.

2009 «Sur les catégories juridiques de crime contre l'humanité et de génocide», in I. Delpla \& M. Bessone (dir.), Peines de guerre. La justice pénale internationale et l'ex-Yougoslavie, Paris, Éditions de l'EHESS : 53-66.

LEWIS, D. A.

2009 «Unrecognized Victims : Sexual Violence Against Men in Conflict Settings Under International Law », Wisconsin International Law Journal, 27 (1) : $1-49$.

MÉDECINS SANS FRONTIÈRES (MSF)

2009 Shattered Lives. Immediate Medical Care Vital for Sexual Violence Victims, Bruxelles, MSF.

MOOKHERJEe, N.

2011 «"Héroïnes de guerre" et hommes oubliés de la guerre de libération du Bangladesh », in R. BRANCHE \& F. VIRGILI (dir.), op. cit. : 71-81.

SARTRE, J.-P.

1964 «Une victoire », in Situations, V, Paris, Gallimard : 72-88 (« Une victoire », L'Express, 350, 6 mars 1958).

SivaKumaran, S.

2005 «Male/Male Rape and the "Taint" of Homosexuality », Human Rights Quarterly, 27 (4) : 1274-1306.

2007 «Sexual Violence Against Men in Armed Conflict», The European Journal of International Law, 18 (2) : 253-276. 
STEMPLE, L.

2009 «Male Rape and Human Rights », Hastings Law Journal, 60 (3) : 605-646.

Stener Carlson, E.

1997 «Sexual Assault on Men in War», The Lancet, 340 (9045) : 129.

STORR, W.

2011 «The Rape of Men », The Observer, July 17. $<$ http://www.guardian.co.uk/society/2011/jul/17/the-rape-of-men>.

\section{RÉSUMÉ}

Les violences sexuelles commises contre des hommes au cours de conflits armés ont longtemps été négligées. Elles ont été reconnues par des activistes des droits de I'homme et quelques ONG médicales dans les contextes de guerre en ex-Yougoslavie et à I'Est de la République du Congo. Puis, au début des années 2000, des études ont commencé à se donner pour objectif à la fois d'enquêter sur ces formes de brutalité et d'expliquer le fait qu'elles aient été si rarement considérées par les ONG et les agences des Nations Unies. Nous examinons ces études et les explications qu'elles donnent aux approches exclusivement orientées sur les viols de femmes.

\section{ABSTRACT}

Male Rape. Masculinities and Armed Conflicts. - For a long time the topic of sexual violence against men in wartime has been neglected. Inquiries have been conducted for the first time by human rights activists and some medical NGOS during wars in ex-Yugoslavia and the Democratic Republic of Congo. Since as early as 2000, researchers began to investigate sexual assaults on men, and at the same time tried to explain why general comments by NGOS and UN agencies about sexual violence have explicitly excluded male victims. We examine these studies and the critical explanations they give for approaches exclusively oriented on the rape of women.

Mots-clés/keywords : République démocratique du Congo, conflits armés, droit international, homophobie, violence sexuelle, viols d'hommes/Democratic Republic of Congo, armed conflicts, international law, homophobia, sexual violence, male rape. 